\title{
Tracing the stellar halo of an early type galaxy out to 25 effective radii
}

\author{
Marina Rejkuba ${ }^{1}$ \\ ${ }^{1}$ European Southern Observatory, \\ Karl-Schwarzschild-Strasse 2, 85748 Garching bei München, Germany \\ email: mrejkuba@eso.org
}

\begin{abstract}
We have used ACS and WFC3 cameras on board HST to resolve stars in the halo of NGC 5128 out to $140 \mathrm{kpc}$ (25 effective radii, $\left.R_{\text {eff }}\right)$ along the major axis and $70 \mathrm{kpc}(13$ $R_{\text {eff }}$ ) along the minor axis. This dataset provides an unprecedented radial coverage of stellar halo properties in any galaxy. Color-magnitude diagrams clearly reveal the presence of the red giant branch stars belonging to the halo of NGC 5128 even in the most distant fields. The V-I colors of the red giants enable us to measure the metallicity distribution in each field and so map the metallicity gradient over the sampled area. The stellar metallicity follows a shallow gradient and even out at $140 \mathrm{kpc}\left(25 R_{\text {eff }}\right)$ its median value does not go below $[\mathrm{M} / \mathrm{H}] \sim-1$ dex. We observe significant field-to-field metallicity and stellar density variations. The star counts are higher along the major axis when compared to minor axis field located $90 \mathrm{kpc}$ from the galaxy centre, indicating flattening in the outer halo. These observational results provide new important constraints for the assembly history of the halo and the formation of this gE galaxy.
\end{abstract}

Keywords. galaxies: elliptical and lenticular; galaxies: halos; galaxies: individual (NGC 5128)

\section{Introduction}

Extended galactic components contain important clues for the formation and evolution of large galaxies. While the halos contain relatively small fraction of stellar mass, the properties of stars in the remote halo regions can be used to trace the star formation and assembly history of galaxies. This is a rapidly changing field of research that has benefitted from the new wide field cameras on large ground-based telescopes and from the HST. In this contribution I present the results of our study of the extended stellar halo of NGC 5128 (Centaurus A).

Table 1 lists all early type galaxies with resolved stellar population studies from literature. The closest and easiest to observe among them is NGC 5128, located at a distance of only 3.8 Mpc (Harris et al. 2010). Thanks to its vicinity this galaxy became a Rosetta stone for early-type galaxy halo studies (Harris et al. 1999; Harris \& Harris 2000, 2002; Ferrarese et al. 2007; Rejkuba et al. 2003, 2005, 2011, 2014; Crnojević et al. 2014; Bird et al. 2015). The global galaxy characteristics are representative of other galaxies of its class with the optical luminosity $M_{V}=-21.5$, mass $1.2 \times 10^{12} \mathrm{M}_{\odot}$ (Peng et al. 2004), and the presence of an AGN and supermassive black hole in its center (Neumayer 2010). The fact that this galaxy presents clear evidence of a recent merger is consistent with the fact that $73 \%$ of nearby luminous ellipticals show tidal disturbance (Tal et al. 2009).

\section{NGC 5128 stellar halo studies}

In a series of HST studies, starting with the WFPC2 camera, following with the ACS and most recently with both ACS and WFC3 cameras in parallel, stellar population content in 9 different locations in the halo of NGC 5128 (Fig. 1) was investigated. 
Table 1. Early type galaxies with stellar halo properties studied through resolved stellar population observations.

\begin{tabular}{|c|c|c|c|c|c|c|}
\hline Name & Type & $\begin{array}{c}\mathbf{M}_{V} \\
(\mathrm{mag})\end{array}$ & $\begin{array}{c}(\mathbf{m}-\mathbf{M})_{0} \\
(\mathrm{mag})\end{array}$ & $\begin{array}{l}\text { Distance } \\
(\mathrm{Mpc})\end{array}$ & Environment & $\begin{array}{l}\text { Literature } \\
\text { References }\end{array}$ \\
\hline Maffei 1 & $\mathrm{E}$ & -21.6 & 27.7 & 3.4 & $\begin{array}{l}\text { Maffei/IC342 group } \\
\left(A_{V} \sim 5.1\right)\end{array}$ & $1,2,3$ \\
\hline $\begin{array}{l}\text { NGC } 5128 \\
=\text { Cen } \mathrm{A}\end{array}$ & $\begin{array}{l}\text { E/S0 pec; } \\
\text { Sy2 }\end{array}$ & -21.5 & 27.91 & 3.8 & $\begin{array}{l}\text { Centaurus A } \\
\text { group }\end{array}$ & $4-14$ \\
\hline NGC 3115 & S0 & -21.1 & 30.05 & 10.2 & NGC 3115 group & 15,16 \\
\hline $\begin{array}{l}\text { NGC } 3379 \\
=\text { M } 105\end{array}$ & $\mathrm{E} 1$ & -20.9 & 30.06 & 10.2 & Leo I group & $17-19$ \\
\hline NGC 3377 & E5 & -20.0 & 30.17 & 10.8 & Leo I group & 20 \\
\hline $\begin{array}{l}\text { NGC } 4486 \\
=\mathrm{M} 87\end{array}$ & $\begin{array}{l}\text { E0 pec; } \\
\text { Sy; cD }\end{array}$ & -22.5 & 31.08 & 16.4 & $\begin{array}{l}\text { Virgo cluster } \\
\text { core }\end{array}$ & 21 \\
\hline
\end{tabular}

Literature References: (1) Davidge \& van den Bergh (2001), (2) Davidge (2002), (3) Wu et al. (2014), (4) Soria et al. (1996), (5) Harris et al. (1999), (6) Harris \& Harris (2000), (7) Harris \& Harris (2002), (8) Ferrarese et al. (2007), (9) Rejkuba et al. (2003), (10) Rejkuba et al. (2005), (11) Rejkuba et al. (2011), (12) Rejkuba et al. (2014), (13) Crnojević et al. (2013), (14) Bird et al. (2015), (15) Elson (1997), (16) Peacock et al. (2015), (17) Sakai et al. (1997), (18) Gregg et al. (2004), (19) Harris et al. (2007b), (20) Harris et al. (2007a), (21) Bird et al. (2010)

The three WFPC2 fields, F1, F2 and F3, probed the inner halo at projected distances of 8,21 and $31 \mathrm{kpc}$ (equivalent to 1.5, 4 and $5.5 \mathrm{R}_{\mathrm{eff}}$ ) and established that the halo stars have magnitudes and colours consistent with the red giant branch (RGB) stars spanning a wide range of metallicities. Adopting an empirical calibration of $(V-I)$ vs. $[\mathrm{M} / \mathrm{H}]$ (colour vs. metallicity) based on $(V-I)$ RGB fiducial lines for Galactic globular clusters with spectroscopically measured metallicities, the first metallicity distribution function (MDF) for stars in a halo of an early type galaxy was determined. It showed an asymmetric distribution that peaks around -0.5 dex and has a long tail towards the metal-poor end (Harris et al. 1999, Harris \& Harris 2000, 2002).

The ACS data in F4, at projected distance of $38 \mathrm{kpc}\left(7 \mathrm{R}_{\mathrm{eff}}\right)$ are the deepest observations of an early type galaxy, reaching the core-helium burning red clump evolutionary phase (Rejkuba et al. 2005). A comparison of the observed vs. simulated colourmagnitude diagrams (CMDs) and luminosity functions showed that $70-80 \%$ of the halo stars formed $12 \pm 1$ Gyr ago already spanning the entire range of model metallicities used in simulations $(\mathrm{Z}=0.0001-0.04)$. The $20-30 \%$ of the younger stars were best fitted with the models having higher minimum metallicity $\left(\sim 1 / 10-1 / 4 Z_{\odot}\right)$ and ages between $2-4$ Gyr. In other words, the bulk of the halo stars in NGC 5128 formed at redshift $z \gtrsim 2$ and the chemical enrichment was very fast, reaching solar or even twice-solar metallicity already 11-12 Gyr ago, while the minor younger component, contributing $\sim 10 \%$ of the halo mass formed 2-4 Gyr ago (Rejkuba et al. 2011).

The aim of the latest study, which used ACS and WFC3 cameras in parallel, was to address the question how far the halo extends and to measure the metallicity gradients along the minor and major axis of the outer galaxy halo (Rejkuba et al. 2014). Two minor axis locations, F8 at $40 \mathrm{kpc}$ and F9 at $90 \mathrm{kpc}$ were observed, while 3 fields were placed along the major axis: F5 at $60 \mathrm{kpc}$ projected distance, F6 at $90 \mathrm{kpc}$, and F7 at $140 \mathrm{kpc}$. The expectation was that the remote location, $140 \mathrm{kpc}$ away $\left(25 \mathrm{R}_{\mathrm{eff}}\right)$, of $\mathrm{F} 7 \mathrm{might}$ be used as background/foreground control field if no significant RGB population belonging to NGC 5128 halo is identified, while the F5 location at $11 \mathrm{R}_{\text {eff }}$ was selected close to expected transition distance between the inner and outer halo, based on observed halo 


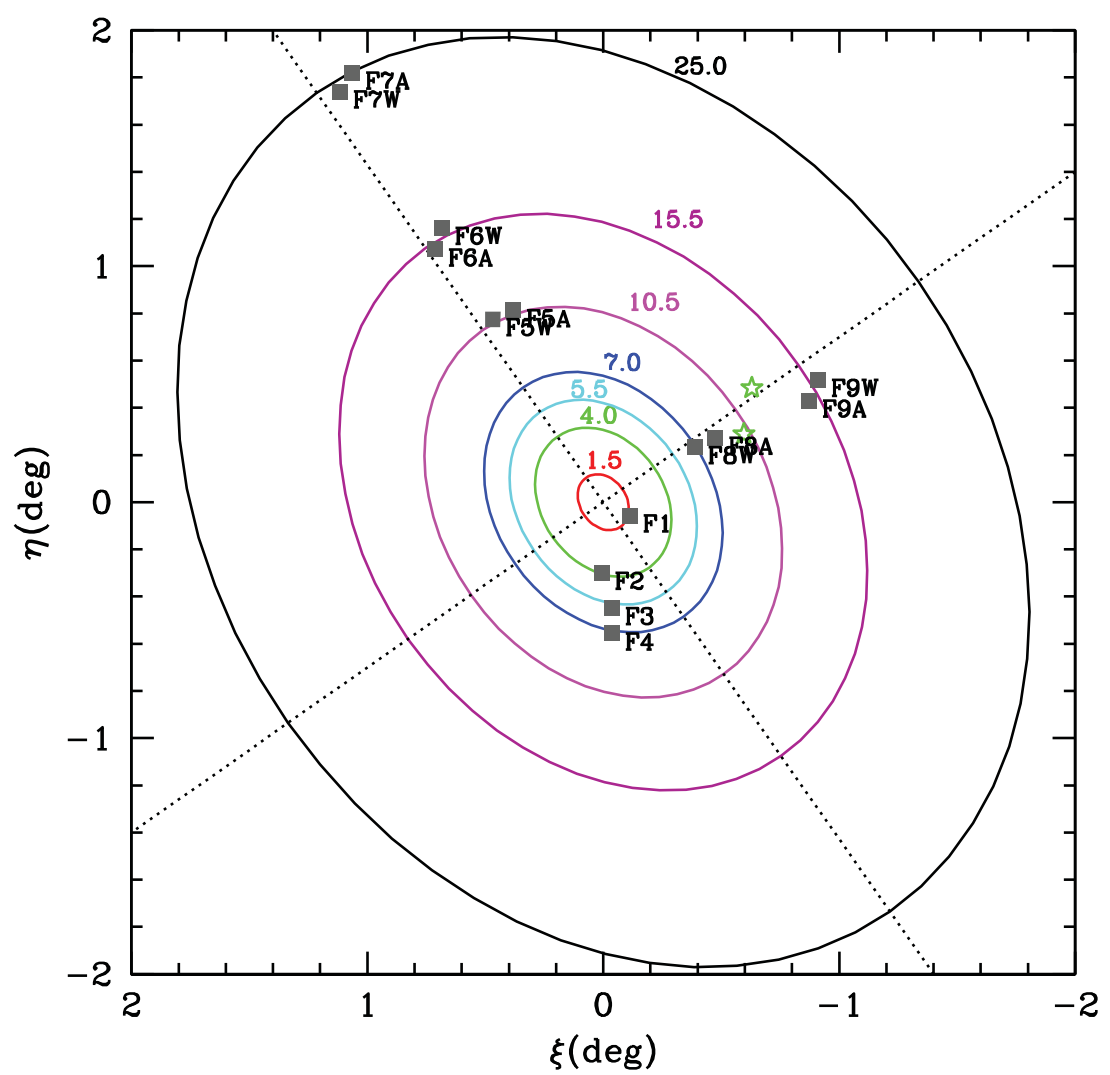

Figure 1. Distribution of HST studies in the halo of NGC 5128 relative to the galaxy centre. The elliptical contours with an axis ratio of 0.77 and $\mathrm{PA}=35^{\circ}$, measured from the galaxy light profile in the inner $2 R_{\text {eff }}$, indicate distance from the centre in units of effective radii $\left(R_{\text {eff }}\right)$.

density profile break in NGC 3379 (Harris et al. 2007b). The location of F8 was along the same projected elliptical radius $\left(7 \mathrm{R}_{\text {eff }}\right)$ as $\mathrm{F} 4$, while the minor and major axis fields F9 and F6 were located along the ellipse corresponding to the extrapolation to $15.5 \mathrm{R}_{\text {eff }}$. These were chosen to facilitate the comparison of the stellar density profiles along the minor and major axis and thus the overall halo shape.

\section{Removing the foreground Milky Way contamination}

The relative fraction of the Milky Way (MW) foreground vs. RGB stars in NGC 5128 becomes important, as the density of halo decreases. At some point the density of upper RGB stars in the halo becomes comparable to that of the MW stars and it is impossible to further trace the NGC 5128 halo. Hence, to establish the galaxy and halo extent, it is important to accurately subtract the MW contamination from the observed CMDs.

As a first step to estimate the foreground contamination TRILEGAL (Girardi et al. 2005) and Besançon (Robin et al. 2003) MW models were examined. The foreground contamination by MW stars consists mainly of dwarf stars in the thin, thick disk, and the halo according to these models. Rejkuba et al. (2014) found large differences between the two models: Besançon simulations yielded more than 3 times fewer stars, in particular having fewer faint stars than TRILEGAL, while their colour distribution better fit the observed distribution of MW stars brighter than the tip of the RGB. However, since 

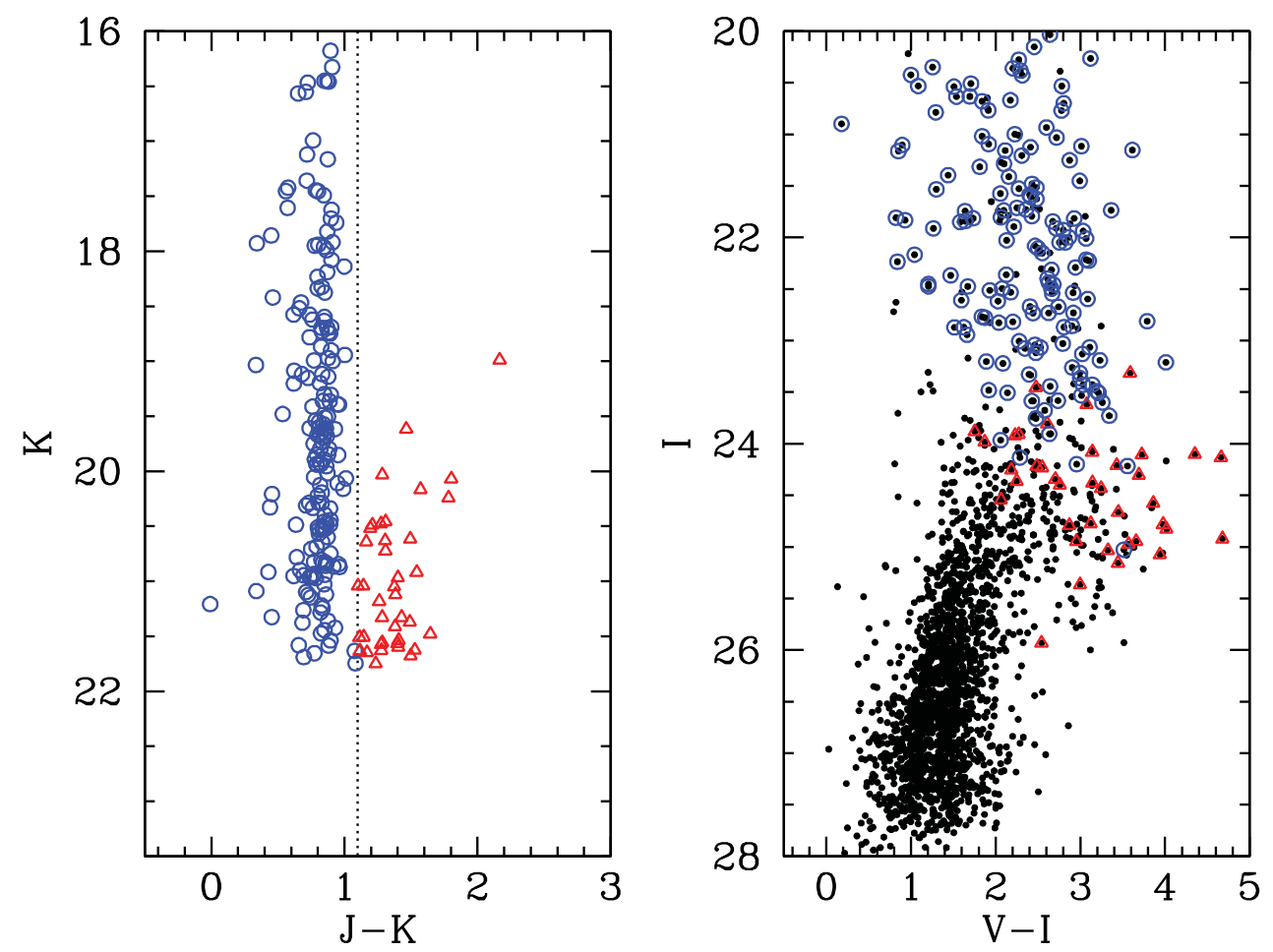

Figure 2. Left: F5 $J K_{s}$ CMD based on PSF photometry of stars detected on ISAAC images. Stars bluer than $J-K_{s}<1.1$ (blue circles) are likely MW foreground contaminants, while the redder stars (red triangles) belong to NGC 5128 halo. Right: VI CMD of the same area (F5) based on WFC3 data. The stars found in common on the ISAAC images are shown as blue or red dots according to their $J-K_{s}$ colour measured from ISAAC data.

TRILEGAL colour distribution well matched the observed one for fainter $(I>24.5)$ part of the CMD, where most of the NGC 5128 halo stars are located, and given that it had higher number of stars, this simulation was preferred in order to avoid underestimating the MW contamination.

The number of stars observed in the RGB area of the CMD in F7 at $140 \mathrm{kpc}$ distance is 2.14 times higher than the number of expected MW stars according to the TRILEGAL simulation, demonstrating that the NGC 5128 halo extends beyond the most distant field observed. After statistical decontamination of the observed CMDs using TRILEGAL MW model, the RGB colours were interpolated on a grid of empirical RGB fiducial lines in order to derive MDF in each observed field (F5-F9), for ACS and WFC3 fields independently. In each field the mean and median metallicity and stellar density were computed - these profiles are presented in the next section.

Before discussing the resulting gradients (constructed based on foreground decontamination with models), an alternative, purely empirical, way to decontaminate the CMDs is presented. It is based on combination of near-IR and optical data. ISAAC camera on the ESO VLT was used to obtain $J$ and $K_{s}$ images coinciding with the WFC3 observations of F5-F9 fields. The size of the ISAAC field of view matches very well that of the WFC3. Fig. 2 shows on the left the $J-K_{s}$ vs. $K_{s}$ CMD constructed with the PSF photometry of stellar sources on the ISAAC images of F5. On the right the ISAAC detections in common with the ones from WFC3 are overplotted on the WFC3 VI CMD. Stars detected on ISAAC images with $J-K_{s}<1.1$ (blue circles) most likely belong to 

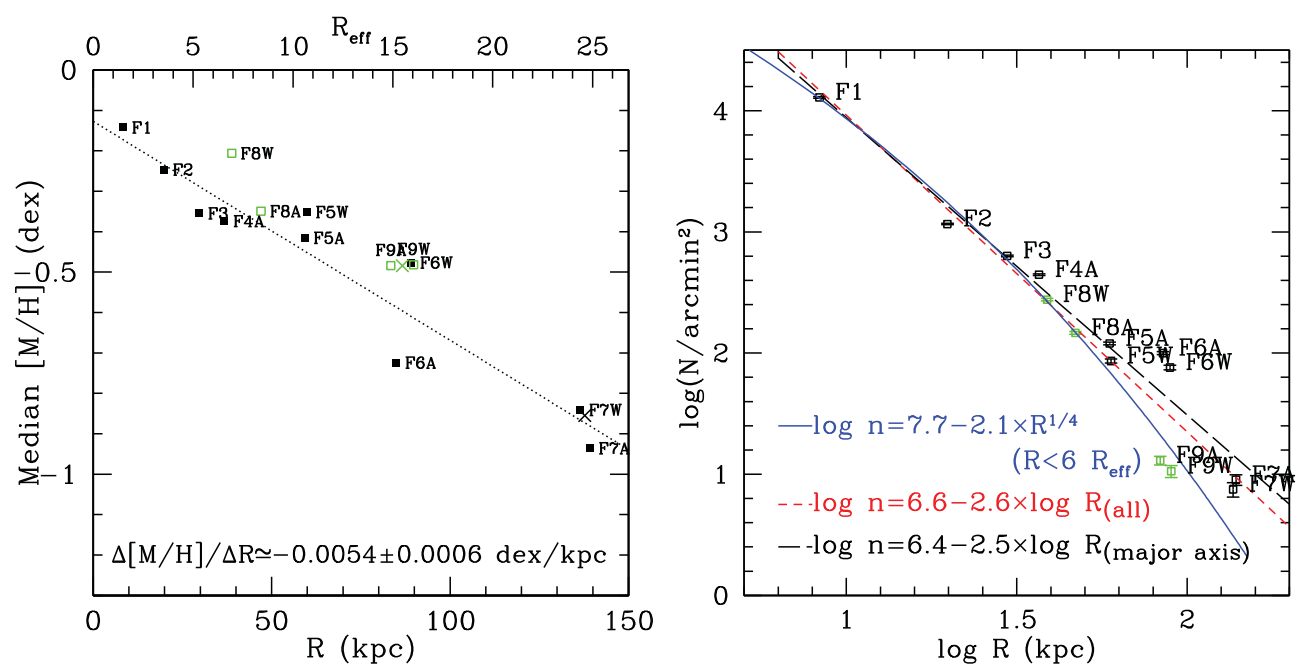

Figure 3. Left: median metallicity gradient. Right: stellar density gradient. The measurements in the minor axis fields (F8 and F9) are shown with the green open symbols and their distances are scaled to the major axis by multiplying with the inverse axis ratio $(\mathrm{a} / \mathrm{b}=1 / 0.77)$.

the MW foreground population, while those redder are likely members of the NGC 5128 halo. This figure shows that $J-K_{s}$ colour can be used to effectively decontaminate the CMDs above the RGB tip and therefore investigate a possible presence of bright AGB stars. At fainter magnitudes it is also possible to get $J-K_{s}$ colours by adopting positions of stars from WFC3 data. These measurements are somewhat noisier and the separation between foreground and NGC 5128 halo stars is not as clean (Rejkuba et al., in prep).

\section{Results and Conclusions}

Based on the observed HST CMDs of fields F1-F9, from which the MW foreground contamination was statistically removed, the mean metallicity, metallicity dispersion and stellar density was obtained from the upper RGB, where observations do not suffer from incompleteness. In all fields the metallicity distribution was obtained in a homogeneous way by interpolating the $V-I$ colours on a grid of $\alpha$-enhanced BASTI isochrones (Pietrinferni et al. 2006). While the absolute metallicity scale may depend on the selected set of stellar evolutionary models, the relative metallicity between different fields is robust given the uniform measurement procedure. The MDFs for fields within $\sim 15 \mathrm{R}_{\text {eff }}$ distance are similar to that of F4 (Rejkuba et al. (2005)), presenting a single peak, quick drop on the metal-rich side, and long, less populated metal-poor tail. The median metallicity gradient is shown on the left panel in Figure 3 and its slope $\Delta[\mathrm{M} / \mathrm{H}] / \Delta \mathrm{R}=-0.0054 \pm 0.0006$ $\mathrm{dex} / \mathrm{kpc}$ is obtained from a linear fit to the data. The minor axis fields have on average higher metallicity than the corresponding fields along the major axis. However, similar size field-to-field scatter is also observed. The gradient is quite shallow, such that even in the most remote field in the halo, at $140 \mathrm{kpc}\left(25 R_{\text {eff }}\right)$ distance from the galaxy centre, the median metallicity is close to or higher than $[\mathrm{M} / \mathrm{H}]=-1$ dex.

The stellar density gradient is shown in the right panel of Fig. 3. Already from the inspection of the observed CMDs it was obvious that there is a larger density along the major axis - this is confirmed by the star counts. The outer halo density along the minor axis is consistent with the extrapolation of the $\mathrm{R}^{-1 / 4}$ profile fitted to the inner three fields, while the major axis fields present an excess of stars and their density gradient is 
well fitted with a power law. The increasing ellipticity in the outer halo of NGC 5128 confirms a tentative result from the ground based study of Crnojević et al. (2013). The halo extends along the major axis at least to $140 \mathrm{kpc}$ and likely also beyond. To find out how far it extends and determine its shape further observations are needed.

So far only a handful of galaxies have been investigated and their halo coverage is very uneven (Tab. 1$)$. In all of them quite metal-rich $([\mathrm{M} / \mathrm{H}]>-1$ dex) mean (median) metallicity is measured that is unlike the mean metallicity of the MW halo. In NGC 3379 a transition from the centrally concentrated metal-rich to a more extended metal-poor halo is detected beyond $\sim 12 \mathrm{R}_{\text {eff }}$ (Harris et al. 2007b). The galaxy with the most extended coverage, after that of NGC 5128, is NGC 3115. It shows a similarly shallow metallicity gradient, and Peacock et al. (2015) estimated that the metal-poor component $([\mathrm{M} / \mathrm{H}]<-1.3$ dex $)$ would only start dominating over the metal-rich one beyond $\sim 200 \mathrm{kpc}$. No galaxy halo was however traced that far. The future facilities, such as JWST and WFIRST will enable deep and wide area mapping of the extended stellar halos in 100s of galaxies up to $10 \mathrm{Mpc}$, opening the possibility for statistical comparison of their properties with the models (see the review by Johnston in this volume, and references therein).

\section{References}

Bird, S., Harris, W. E., Blakeslee, J. P., \& Flynn, C. 2010, A $8 A$ 524, A71

Bird, S., Flynn, C., Harris, W. E., \& Valtonen, M. 2015, A\&A 575, A72

Crnojević, D., Ferguson, A. M. N., \& Irwin, M. J. 2013, MNRAS 432, 832

Davidge, T. J. 2002, AJ 124, 2012

Davidge, T. J. \& van den Bergh, S. 2001, ApJ (Letters) 553, L133

Elson, R.A.W. 1997, MNRAS 286, 771

Ferrarese, L., Mould, J. R., \& Stetson, P. B. 2007, ApJ 654, 186

Girardi, L., Groenewegen, M. A. T., Hatziminaoglou, E., \& da Costa, L. 2005, A\&A A 436, 895

Gregg, M. D., Ferguson, H. C., Minniti, D., et al. 2004, AJ, 127, 1441

Harris, G. L. H., Harris, W. E., \& Poole, G. B. 1999, AJ 117, 855

Harris, G. L. H. \& Harris, W. E. 2000 AJ 120, 2423

Harris, G. L. H., Rejkuba, M., \& Harris, W. E. 2010, PASA 27, 475

Harris, W. E. \& Harris, G. L. H. 2002, AJ 123, 3108

Harris, W. E., Harris, G. L. H., Layden, A. C., \& Stetson, P. B. 2007a, AJ 134, 43

Harris, W. E., Harris, G. L. H., Layden, A. C., \& Wehner, E. M. H. 2007b, ApJ 666, 903

Neumayer, N. 2010, PASA 27, 449

Peacock, M. B., Strader, J, Romanowsky, A. J., \& Brodie, J. P. 2015, ApJ 800, 13

Peng, E. W., Ford., H. C. \& Freeman, K. C. 2004, ApJ 602, 685

Pietrinferni, A., Cassisi, S., Salaris, M., \& Castelli, F. 2006, ApJ 642, 797

Rejkuba, M., Minniti, D., Silva, D. R., \& Bedding, T. 2003, A $\mathscr{G} A 411,351$

Rejkuba, M., Harris, W. E., Greggio, L., et al. 2005, ApJ 631, 262

Rejkuba, M., Greggio, L., Harris, W. E., \& Harris, G. L. H. 2011, A\&A 526, A123

Rejkuba, M., Harris, W. E., Greggio, L., et al. 2014, ApJ (Letters) 791, L2

Robin, A. C., Reylé, C., Derriére, S., \& Picaud, S. 2003, A\& A, 409, 523

Sakai, S., Madore, B. F., Freedman, W. L., et al. 1997, ApJ 478, 49

Soria, R., Mould, J. R., Watson, A. M., et al. 1996, ApJ 465, 79

Tal, T., et al. 2009, AJ 138, 1417

Wu, P-F., Tully, R. B., Rizzi, L. et al. 2014, AJ 148, 7 